\title{
Additive Methods for the Numerical Solution of Ordinary Differential Equations
}

\author{
By G. J. Cooper and A. Sayfy
}

\begin{abstract}
Consider a system of differential equations $x^{\prime}=f(x)$. Most methods for the numerical solution of such a system may be characterized by a pair of matrices $(A, B)$ and make no special use of any structure inherent in the system. In this article, methods which are characterized by a triple of matrices $\left(A ; B_{1}, B_{2}\right)$ are considered. These methods are applied in an additive fashion to a decomposition $f=$ $f_{1}+f_{2}$ and some methods have pronounced advantages when one term of the decomposition is linear. This article obtains algebraic conditions which give the order of convergence of such methods. Some simple examples are displayed.
\end{abstract}

1. Introduction. Consider an initial value problem for a system of $n$ differential equations,

$$
x^{\prime}=f(x), \quad x\left(t_{0}\right)=x_{0} .
$$

Butcher [1] showed that many methods for the numerical solution of the initial value problem may be characterized by a pair of matrices $(A, B)$. Such methods make no special allowance for any structure in the differential system, although in many cases the system occurs naturally in a form where $f=f_{1}+f_{2}$, and frequently one term in this decomposition is linear. To take account of such structure, this article examines certain methods characterized by a triple of matrices $\left(A ; B_{1}, B_{2}\right)$. These methods are used in an additive fashion with a decomposition $f=f_{1}+f_{2}$, which may be time dependent. Since the results extend to methods characterized by $r+1$ matrices $\left(A ; B_{1}, B_{2}, \ldots, B_{r}\right)$, used with a decomposition $f=f_{1}+f_{2}+\cdots+f_{r}$, it is possible to approximate each equation in the differential system in a different way. For example, special methods for certain high order differential equations may be interpreted as additive methods used with a particular decomposition. In this article, a general decomposition is treated. An alternative approach was adopted by Lawson [3]. Lawson considered a decomposition $f=f_{1}+f_{2}$ with $f_{1}$ linear and integrated the linear term before applying a numerical method to the differential system.

To indicate possible advantages in the use of additive methods, consider the trapezoidal rule used with a step length $h$. This gives

$$
y^{(m)}=y^{(m-1)}+\frac{h}{2} f\left(y^{m-1)}\right)+\frac{h}{2} f\left(y^{(m)}\right), \quad m=1,2,3, \ldots,
$$

where $y^{(m)}$ is an approximation to $x\left(t_{m}\right)$, with $t_{m}=t_{0}+m h, m=0,1,2, \ldots$ In

Received January 31, 1978; revised January 3, 1980.

1980 Mathematics Subject Classification. Primary 65 L05. 
general, each step requires the solution of a set of $n$ nonlinear equations. Now consider a decomposition $f=f_{1}+f_{2}$. Then the additive method defined by

$$
\begin{aligned}
y^{(m)}= & y^{(m-1)}+\frac{h}{2} f_{1}\left(y^{(m-1)}\right)+\frac{h}{2} f_{1}\left(y^{(m)}\right) \\
& +h f_{2}\left(y^{(m-1)}+\frac{h}{2} f\left(y^{(m-1)}\right)\right), \quad m=1,2,3, \ldots,
\end{aligned}
$$

is also a second order method. When $f_{1}$ is linear, this method is linearly implicit, requiring the solution of a set of $n$ linear equations in each step. Although a different decomposition may be used for each step, there is a substantial computational gain when the same decomposition is used for several steps. In addition, the method possesses some desirable stability features akin to those enjoyed by the trapezoidal rule. Thus, certain additive methods may be suitable for stiff systems of differential equations.

This particular additive method may be reformulated as the three-stage method given by

$$
\begin{aligned}
& y_{1}^{(m)}=y_{3}^{(m-1)}, \\
& y_{2}^{(m)}=y_{3}^{(m-1)}+\frac{h}{2} f_{1}\left(y_{1}^{(m)}\right)+\frac{h}{2} f_{2}\left(y_{1}^{(m)}\right), \\
& y_{3}^{(m)}=y_{3}^{(m-1)}+\frac{h}{2} f_{1}\left(y_{1}^{(m)}\right)+\frac{h}{2} f_{1}\left(y_{3}^{(m)}\right)+h f_{2}\left(y_{2}^{(m)}\right),
\end{aligned}
$$

for $m=1,2,3, \ldots$ For a fixed step length $h$, a sequence of decompositions $\left\{f=f_{1}^{(m)}+f_{2}^{(m)}\right\}$ may be used. Thus, a general $s$-stage additive method may be formulated as

$$
y_{i}^{(m)}=\sum_{j=1}^{s} a_{i j} y_{j}^{(m-1)}+h \sum_{j=1}^{s} b_{i j} f_{1}^{(m)}\left(y_{j}^{(m)}\right)+h \sum_{j=1}^{s} \beta_{i j} f_{2}^{(m)}\left(y_{j}^{(m)}\right),
$$

for $i=1,2, \ldots, s$ and $m=1,2,3, \ldots$ Here $y_{i}^{(m)}$ may be interpreted as an approximation to $x\left(t_{m-1}-h+c_{i} h\right), i=1,2, \ldots, s, m=0,1,2, \ldots$, where $\mathrm{c}=$ $\left(c_{1}, c_{2}, \ldots, c_{s}\right)^{T}$ is some (consistency) vector. Often it happens that

$$
c_{i}=\sum_{j=1}^{s} b_{i j}=\sum_{j=1}^{s} \beta_{i j}, \quad i=1,2, \ldots, s .
$$

Such a method is described as an $s$-stage additive $\left(A ; B_{1}, B_{2}\right)$ method, where $A=$ $\left\{a_{i j}\right\}, B_{1}=\left\{b_{i j}\right\}$, and $B_{2}=\left\{\beta_{i j}\right\}$, are $s \times s$ matrices. Since it is possible to choose $f_{1}^{(m)}=0$ or $f_{2}^{(m)}=0$ for $m=1,2,3, \ldots$, an additive $\left(A ; B_{1}, B_{2}\right)$ method gives both an $\left(A, B_{1}\right)$ method and an $\left(A, B_{2}\right)$ method as defined by Butcher [1].

A case of special interest arises when $\left\{f_{1}^{(m)}\right\}$ is a sequence of linear maps of $R^{n}$ into $R^{n}$. Then it is appropriate to choose the method $\left(A, B_{1}\right)$ to be semiexplicit so that $B_{1}$ is a lower triangular matrix, and to choose the method $\left(A, B_{2}\right)$ to be explicit so that $B_{2}$ is a strictly lower triangular matrix. The additive method is then linearly implicit, since each step requires the solution of a set of $n$ linear equations for each nonzero diagonal element of $B_{1}$. There is a substantial computational gain if these elements can be chosen equal.

Now assume that $B_{1} \mathrm{e}=B_{2} \mathrm{e}$, where $\mathrm{e}=(1,1, \ldots, 1)^{T}$. Then, it happens that the method may be used to solve a nonautonomous initial value problem $x^{\prime}=f(t, x)$, 
$x\left(t_{0}\right)=x_{0}$, with a fixed step length $h$ and a sequence of decompositions $\left\{f=f_{1}^{(m)}+\right.$ $\left.f_{2}^{(m)}\right\}$. In this case the method is applied in the form

$$
\begin{aligned}
y_{i}^{(m)}= & \sum_{j=1}^{s} a_{i j} y_{j}^{(m-1)}+h \sum_{j=1}^{s} b_{i j} f_{1}^{(m)}\left(t_{m-1}+h c_{j}, y_{j}^{(m)}\right) \\
& +h \sum_{j=1}^{s} \beta_{i j} f_{2}^{(m)}\left(t_{m-1}+h c_{j}, y_{j}^{(m)}\right)
\end{aligned}
$$

for $i=1,2, \ldots, s$, where $t_{m}=t_{0}+m h, m=1,2,3, \ldots$ Again suppose that the additive method is chosen so that the method $\left(A, B_{1}\right)$ is semiexplicit and the method $\left(A, B_{2}\right)$ is explicit. For each $t$, let $\left\{f_{1}^{(m)}\right\}$ be a sequence of linear maps of $R^{n}$ into $R^{n}$. Then the additive method is linearly implicit. Again there is a substantial computational gain if the nonzero diagonal elements of $B_{1}$ can be chosen equal and the corresponding elements of $c$ can be chosen equal also.

Although examples are given, this article concentrates on establishing algebraic conditions which give the order of convergence of additive methods. A treatment of certain stability features, and the derivation of special methods, is deferred to another article. Recently, one of the authors [2] used an order vector to define a sequence of norms and hence established conditions giving the order of convergence of an $(A, B)$ method. Here, this theory is adapted to cope with additive methods, although the type of method considered is restricted in order to simplify the analysis. Instead, it would be possible to adopt the theory developed by Skeel [4]. Skeel points out that the results are applicable when different numerical methods are applied to different equations in the differential system, and the theory may be applied also to additive methods. However, the order of convergence result requires the minimal polynomial of $A$ to have only one zero of modulus one. This is not an intrinsic property of the methods considered here. Further, the theory developed here leads more naturally to algebraic conditions for the order of convergence of additive methods. For these methods it is possible to obtain algebraic conditions which are independent of the decomposition used and which have a simple interpretation in terms of the order conditions for $(A, B)$ methods.

A number of assumptions, concerning the initial value problem and the decompositions, are stated now. These are stronger than necessary in order to simplify the presentation. A more detailed treatment is given by Cooper [2]. Suppose that the initial value problem has a unique analytic solution $x: J \rightarrow R^{n}$, where $[0,1]$ is contained in the open interval $J$, and suppose that $t_{0}=0$ and that solution approximations are required on $[0,1]$. Let $f$ satisfy a Lipschitz condition

$$
\|f(y)-f(z)\| \leqslant L\|y-z\| \quad \forall y, z \in R^{n}
$$

for a given norm on $R^{n}$. Assume that $f$ is a continuously differentiable mapping with derivative $f^{\prime}: R^{n} \rightarrow L\left(R^{n}, R^{n}\right)$, where $L\left(R^{n}, R^{n}\right)$ is the space of continuous linear mappings of $R^{n}$ into $R^{n}$. It is supposed that higher derivatives exist also. The particular mapping $f^{\prime}(x): J \rightarrow L\left(R^{n}, R^{n}\right)$, defined by $f^{\prime}(x)(t)=f^{\prime}(x(t))$, is assumed to be analytic. 
Suppose a step length $h$ is chosen so that $M h=1, M$ a positive integer. Since the decompositions used may depend on the step length and may vary from step to step, it is necessary to consider sequences of decompositions

$$
\left\{f=f_{1 M}^{(m)}+f_{2 M}^{(m)}\right\}_{m=1}^{M}, \quad M=1,2,3, \ldots
$$

It is assumed that the components satisfy Lipschitz conditions

$$
\left\|f_{i M}^{(m)}(y)-f_{i M}^{(m)}(z)\right\| \leqslant L_{i}\|y-z\|, \quad i=1,2, m=1,2, \ldots, M, M=1,2,3, \ldots,
$$

on $R^{n}$. Further, it is assumed that each component has continuous derivatives and that the special mappings

$$
f_{1 M}^{(m)^{\prime}}(x), f_{2 M}^{(m)^{\prime}}(x), \quad m=1,2, \ldots, M, M=1,2,3, \ldots,
$$

are analytic. It is to be understood, throughout, that the definitions refer to an arbitrary initial value problem and to arbitrary sequences of decompositions which satisfy these assumptions.

The $s$-stage methods considered are defined by reference to the space $R^{s}$ with elements $\mathbf{w} \in R^{s}$ interpreted as column vectors $\mathbf{w}=\left(w_{1}, w_{2}, \ldots, w_{s}\right)^{T}$. The elements $\mathrm{e}_{1}, \mathrm{e}_{2}, \ldots, \mathrm{e}_{s}$ are the natural basis for $R^{s}$, and $\mathrm{e}=\mathrm{e}_{1}+\mathrm{e}_{2}+\cdots+\mathrm{e}_{s}$ is the unit element. An order vector is an element $\mathrm{p} \in R^{s}$ with positive integer components $p_{i}=$ $\mathbf{e}_{i}^{T} \mathbf{p}, i=1,2, \ldots, s$. It is supposed that a given method has some order vector, and hence a sequence of norms on $R^{s}$,

$$
\|\mathrm{w}\|_{M}=\max _{1 \leqslant i \leqslant s} M^{p_{i}}\left|w_{i}\right|, \quad M=1,2,3, \ldots
$$

associated with it. (The order vector associated with a method is not unique.) Let $A=\left\{a_{i j}\right\}$ be a real $s \times s$ matrix. Then

$$
\|A\|_{M}=\sup _{\|\mathrm{w}\|_{M} \leqslant 1}\|A \mathrm{w}\|_{M}=\max _{1 \leqslant i \leqslant s} \sum_{j=1}^{s}\left|a_{i j}\right| M^{p_{i}-p_{j}} .
$$

Let $R^{N}=R^{n} \times R^{n} \times \cdots \times R^{n}$ with $N=n s$ so that $Y=y_{1} \oplus y_{2} \oplus \cdots \oplus y_{s}$, where $y_{i} \in R^{n}, i=1,2, \ldots, s$, is an element of $R^{N}$. A sequence of norms on $R^{N}$ is defined by

$$
\|Y\|_{M}=\max _{1 \leqslant i \leqslant s} M^{p_{i}}\left\|y_{i}\right\|, \quad M=1,2,3, \ldots
$$

Any real $s \times s$ matrix $A=\left\{a_{i j}\right\}$ defines a linear map A: $R^{N} \rightarrow R^{N}$,

$$
\mathbf{A} Y=\sum_{j=1}^{s} a_{1 j} y_{j} \oplus \sum_{j=1}^{s} a_{2 j} y_{j} \oplus \cdots \oplus \sum_{j=1}^{s} a_{s j} y_{j},
$$

so that $\mathrm{A}=A \otimes I$ is the tensor product of $A$ and the $n \times n$ identity matrix $I$. It follows that $\|\mathbf{A}\|_{M}=\|A\|_{M}$.

The mapping $f: R^{n} \rightarrow R^{n}$ defines a mapping $F: R^{N} \rightarrow R^{N}$, where $F(Y)=$ $f\left(y_{1}\right) \oplus f\left(y_{2}\right) \oplus \cdots \oplus f\left(y_{s}\right)$. Thus, for each norm, $F$ satisfies a Lipschitz condition on $R^{N}$ with Lipschitz constant $L$. The derivative of $F$ is the mapping $F^{\prime}: R^{N} \rightarrow$ $L\left(R^{N}, R^{N}\right)$, defined by $F^{\prime}(Y) Z=f^{\prime}\left(y_{1}\right) z_{1} \oplus f^{\prime}\left(y_{2}\right) z_{2} \oplus \cdots \oplus f^{\prime}\left(y_{s}\right) z_{s}$. Thus,

$$
\left\|F^{\prime}(Y)\right\|_{M}=\sup _{\|Z\|_{M} \leqslant 1}\left\|F^{\prime}(Y) Z\right\|_{M}=\max _{1 \leqslant i \leqslant s}\left\|f^{\prime}\left(y_{i}\right)\right\| \leqslant L \quad \forall Y \in R^{N} .
$$


Since $x: J \rightarrow R^{n}$ is analytic, the mapping $X: J^{s} \rightarrow R^{N}$, defined by $X(\mathbf{w})=x\left(w_{1}\right) \oplus$ $x\left(w_{2}\right) \oplus \cdots \oplus x\left(w_{s}\right)$, is analytic, as also is the mapping $F^{\prime}(X)$ defined by $F^{\prime}(X)(\mathbf{w})=$ $F^{\prime}(X(\mathrm{w}))$. Let $\mathrm{c}=\left(c_{1}, c_{2}, \ldots, c_{s}\right)^{T}$ and let $C$ be the $s \times s$ diagonal matrix with diagonal elements $c_{i i}=c_{i}, i=1,2, \ldots, s$. Then $\mathrm{C}=C \otimes I$ is a linear map of $R^{N} \rightarrow$ $R^{N}$ and, provided that $\mathrm{w}+\mathrm{c} \in J^{s}$,

$$
\begin{gathered}
X(\mathbf{w}+\mathbf{c})=X(\mathbf{w})+\mathbf{C} X^{\prime}(\mathbf{w})+\cdots+\frac{1}{\tau !} \mathbf{C}^{\tau} X^{(\tau)}(\mathbf{w})+\cdots \\
F^{\prime}(X(\mathbf{w}+\mathbf{c}))=F^{\prime}(X(\mathbf{w}))+\mathbf{C} D F^{\prime}(X(\mathbf{w}))+\cdots+\frac{1}{\tau !} \mathbf{C}^{\tau} D^{\tau} F^{\prime}(X(\mathbf{w}))+\cdots
\end{gathered}
$$

Here, for example, $X^{\prime}(\mathbf{w})=x^{\prime}\left(w_{1}\right) \oplus x^{\prime}\left(w_{2}\right) \oplus \cdots \oplus x^{\prime}\left(w_{s}\right)$ and $D F^{\prime}(X): J^{s} \rightarrow$ $L\left(R^{N}, R^{N}\right)$ is defined by

$$
D F^{\prime}(X)(\mathbf{w}) Y=\frac{d}{d w_{1}} f^{\prime}\left(x\left(w_{1}\right)\right) y_{1} \oplus \frac{d}{d w_{2}} f^{\prime}\left(x\left(w_{2}\right)\right) y_{2} \oplus \cdots \oplus \frac{d}{d w_{s}} f^{\prime}\left(x\left(w_{s}\right)\right) y_{s} .
$$

Sequences of decompositions of $f$ define sequences of decompositions of $F$

$$
\left\{F=F_{1 M}^{(m)}+F_{2 M}^{(m)}\right\}_{m=1}^{M}, \quad M=1,2,3, \ldots,
$$

and the components satisfy properties similar to those described for $F$.

2. The Additive Methods. An $s$-stage additive method is characterized by a triple of real $s \times s$ matrices $\left(A ; B_{1}, B_{2}\right)$ and is associated with sequences of decompositions $\left\{f=f_{1 M}^{(m)}+f_{2 M}^{(m)}\right\}$, where $M=1,2,3, \ldots$ For some $M^{\prime}>0$ the method is defined by

$$
\begin{aligned}
Y_{M}^{(m)}=\mathbf{A} Y_{M}^{(m-1)}+\frac{1}{M} \mathbf{B}_{1} F_{1 M}^{(m)}\left(Y_{M}^{(m)}\right)+\frac{1}{M} \mathbf{B}_{2} F_{2 M}^{(m)}\left(Y_{M}^{(m)}\right) & \\
& m=1,2, \ldots, M, M \geqslant M^{\prime}
\end{aligned}
$$

where $Y_{M}^{(m)}=y_{M 1}^{(m)} \oplus y_{M 2}^{(m)} \oplus \cdots \oplus y_{M s}^{(m)}$ is interpreted as an approximation to

$$
\begin{aligned}
X_{M}^{(m)} & =X\left(\frac{1}{M}(m \mathbf{e}-\mathbf{e}+\mathbf{c})\right) \\
& =x\left(\frac{m-1+c_{1}}{M}\right) \oplus x\left(\frac{m-1+c_{2}}{M}\right) \oplus \cdots \oplus x\left(\frac{m-1+c_{s}}{M}\right),
\end{aligned}
$$

for some $\mathrm{c} \in R^{s}$, for each $m=0,1, \ldots, M$. Thus, for a given step length $h=1 / M$ and a given $Y_{M}^{(0)}$, the method provides approximations on an interval $[a, b] \supset[0,1]$. Equation (2.1) has the form $Y=G(Y)$ with

$$
G(Y)=\mathbf{A} Z+\frac{1}{M} \mathbf{B}_{1} F_{1}(Y)+\frac{1}{M} \mathbf{B}_{2} F_{2}(Y)
$$

where $F_{1}$ and $F_{2}$ satisfy Lipschitz conditions on $R^{N}$ with Lipschitz constants $L_{1}$ and $L_{2}$. It follows that $G$ is a contraction mapping and hence that the sequences $\left\{Y_{M}^{(m)}\right\}$ exist and are unique, provided that $M \geqslant M^{\prime}$, where $M^{\prime}>L_{1}\left\|B_{1}\right\|_{1}+L_{2}\left\|B_{2}\right\|_{1}$.

Motivations for the following definitions have been given by Cooper [2]. It is recalled that the definitions refer to an arbitrary initial value problem with arbitrary sequences of decompositions. It is supposed that a given method has a particular order vector assigned to it. This order vector defines the norms employed. 
Definition 1. An additive $\left(A ; B_{1}, B_{2}\right)$ method is order $\mathbf{p}$ convergent if for each $K>0 \exists K^{\prime}$ and $M^{\prime}$ such that

$$
\left\|X_{M}^{(0)}-Y_{M}^{(0)}\right\|_{M}<K \Rightarrow\left\|X_{M}^{(m)}-Y_{M}^{(m)}\right\|_{M}<K^{\prime}, \quad m=1,2, \ldots, M, \forall M \geqslant M^{\prime} .
$$

By choosing trivial sequences of decompositions, an additive $\left(A ; B_{1}, B_{2}\right)$ method may be reduced to either an $\left(A, B_{1}\right)$ or an $\left(A, B_{2}\right)$ method so that each of these methods must be order $\mathbf{p}$ convergent if the additive method is order $\mathbf{p}$ convergent. In particular, order e convergence of an $(A, B)$ method is equivalent to the definition of convergence given by Butcher [1]. Thus, order e convergence of the additive method implies convergence of the $\left(A, B_{1}\right)$ and $\left(A, B_{2}\right)$ methods.

An $(A, B)$ method is convergent only if it is stable, and a method is defined to be stable if $A$ is power bounded

$$
\left\|A^{\nu}\right\|_{1}<\alpha, \quad \nu=0,1,2, \ldots
$$

Likewise, an additive $\left(A ; B_{1}, B_{2}\right)$ method is defined to be stable if $A$ is power bounded.

To simplify the analysis, this article is restricted to those additive methods which may be considered to belong to a general class of hybrid methods. For a given order vector $\mathrm{p}$, an additive $\left(A ; B_{1}, B_{2}\right)$ method is defined to be hybrid with respect to $\mathrm{p}$ if

$$
p_{j}<p=\max _{1 \leqslant i \leqslant s} p_{i} \Rightarrow A \mathrm{e}_{j}=0, \quad j=1,2, \ldots, s .
$$

This definition depends on the order vector chosen. A method can be both order $\mathbf{p}$ convergent and order $\pi$ convergent but hybrid with respect to $\mathbf{p}$ only. (Conventional hybrid methods are covered by the definition.) A method which is hybrid with respect to $\mathbf{p}$ uses, in a given step, only approximations of 'maximum' order $p$ from the previous step. This has a remarkable effect. Let $H$ be any element of $L\left(R^{N}, R^{N}\right)$. Then

$$
\begin{aligned}
\|H \mathbf{A}\|_{M} & =\sup _{\|Y\|_{M} \leqslant 1}\left\|H\left(\sum_{j=1}^{s} a_{1 j} y_{j} \oplus \sum_{j=1}^{s} a_{2 j} y_{j} \oplus \cdots \oplus \sum_{j=1}^{s} a_{s j} y_{j}\right)\right\|_{M} \\
& =\sup _{\|Z\|_{M} \leqslant 1}\left\|H\left(\sum_{j=1}^{s} a_{1 j} z_{j} \oplus \sum_{j=1}^{s} a_{2 j} z_{j} \oplus \cdots \oplus \sum_{j=1}^{s} a_{s j} z_{j}\right)\right\|_{M},
\end{aligned}
$$

where $z_{j}=0$ if $p_{j}<p$. Thus $\|Z\|_{M}=M^{p}\|Z\|_{1}$. On the other hand $\|W\|_{M} \leqslant M^{p}\|W\|_{1}$ for any $W \in R^{N}$ so that $\|H \mathrm{~A} Z\|_{M} \leqslant M^{p}\|H \mathrm{~A} Z\|_{1}$. Hence, for a hybrid method,

$$
\|H \mathbf{A}\|_{M} \leqslant\|H \mathbf{A}\|_{1} \leqslant\|H\|_{1}\|A\|_{1}, \quad M=1,2,3, \ldots
$$

To define order $\mathbf{p}$ consistency, consider sequences $\left\{Z_{M}^{(m)}\right\}$, where $Z_{M}^{(0)}=X_{M}^{(0)}$ and

$$
\begin{aligned}
Z_{M}^{(m)}=\mathbf{A} X_{M}^{(m-1)}+\frac{1}{M} \mathbf{B}_{1} F_{1 M}^{(m)}\left(Z_{M}^{(m)}\right)+\frac{1}{M} \mathbf{B}_{2} F_{2 M}^{(m)}\left(Z_{M}^{(m)}\right), & \\
m & =1,2, \ldots, M, M \geqslant M^{\prime} .
\end{aligned}
$$

Again, this equation defines a contraction mapping and the sequences $\left\{Z_{M}^{(m)}\right\}$ exist and are uniquely defined for all $M \geqslant M^{\prime}$, where $M^{\prime}>L_{1}\left\|B_{1}\right\|_{1}+L_{2}\left\|B_{2}\right\|_{1}$. The components of $X_{M}^{(m)}-Z_{M}^{(m)}$ give the errors in each stage of step $m$, when the step is started with exact solution values. 
Definition 2. An additive $\left(A ; B_{1}, B_{2}\right)$ method is order $\mathbf{p}$ consistent if $\exists k$ and $M^{\prime}$ and an integer $\omega \geqslant 0$ such that

$$
\begin{aligned}
\left\|X_{M}^{(m)}-Z_{M}^{(m)}\right\|_{M} & <k, \\
\left\|\mathbf{A}^{\omega}\left(X_{M}^{(m)}-Z_{M}^{(m)}\right)\right\|_{M} & <\frac{k}{M},
\end{aligned}
$$

Since the sequence $\left\{A^{M}\right\}$ may not have a limit, it is not possible to employ the definition used by Skeel [4]. A method may also be described as order $p(\omega)$ consistent, and an order $\mathrm{p}(\omega)$ consistent method must be order $\mathrm{e}(\omega)$ consistent. By considering special sequences of decompositions it follows that if an $\left(A ; B_{1}, B_{2}\right)$ method is order $\mathrm{e}(\omega)$ consistent then both the $\left(A, B_{1}\right)$ and $\left(A, B_{2}\right)$ methods are order $\mathrm{e}(\omega)$ consistent. Cooper [2] showed that a stable $(A, B)$ method is order $\mathrm{e}(\omega)$ consistent if and only if it is consistent. Thus, if an $\left(A ; B_{1}, B_{2}\right)$ method is stable and order $\mathrm{e}(\omega)$ consistent, both the $\left(A, B_{1}\right)$ and $\left(A, B_{2}\right)$ methods must be consistent so that $A \mathrm{e}=\mathrm{e}$ and there exist consistency vectors $c_{1}$ and $c_{2}$ such that

$$
A \mathrm{c}_{1}+B_{1} \mathrm{e}=\mathrm{c}_{1}+\mathrm{e}, \quad A \mathrm{c}_{2}+B_{2} \mathrm{e}=\mathrm{c}_{2}+\mathrm{e}
$$

The consistency vectors may be chosen equal if and only if $B_{1} \mathbf{e}=B_{2} \mathrm{e}$, but it is possible to obtain additive methods (for autonomous systems) where $B_{1} \mathrm{e} \neq B_{2} \mathrm{e}$.

Suppose that a stable and order e consistent additive $\left(A ; B_{1}, B_{2}\right)$ method is applied to the initial value problem $x^{\prime}=1$ with $x(0)=0$ using a decomposition $f_{1}=$ $1-\alpha$ and $f_{2}=\alpha$. Since $N=s$, elements of $R^{N}$ may be interpreted as column vectors. Let $Y_{M}^{(0)}=(1 / M)(\mathrm{c}-\mathrm{e})$ for some $\mathrm{c} \in R^{s}$. Then, using the consistency condition (2.4), the method gives

$$
Y_{M}^{(m)}=\frac{1}{M}\{(m-1) \mathbf{e}+\mathbf{c}\}+\frac{1}{M}\left(A^{m}-I\right)\left\{(1-\alpha)\left(\mathbf{c}-\mathbf{c}_{1}\right)+\alpha\left(\mathbf{c}-\mathbf{c}_{2}\right)\right\}
$$

where $I$ is the identity matrix. The method is order e convergent but, for an arbitrary $\alpha$, the initial value problem is integrated exactly if and only if $c-c_{1}$ and $c-c_{2}$ both belong to the null space of $A-I$. This occurs only when $B_{1} \mathrm{e}=B_{2} \mathrm{e}$, and in this case the method may be adapted to handle a nonautonomous problem. When $B_{1} \mathrm{e} \neq B_{2} \mathrm{e}$, a nonautonomous problem should be converted to autonomous form before the method is applied (although it is possible to consider decompositions where the time dependence occurs only in one term of the decomposition).

3. Order of Convergence. In this section, it is shown that a hybrid additive method is order $\mathbf{p}$ convergent if it is stable and order $\mathbf{p}$ consistent. It seems to be necessary to use certain inverse mappings and, in this respect, the argument is similar to that used by Cooper [2]. Partly because hybrid methods alone are considered, the argument given here is shorter and more direct.

THEOREM 1. A hybrid additive $\left(A ; B_{1}, B_{2}\right)$ method is order $\mathbf{p}$ convergent if it is stable and order $\mathrm{p}$ consistent. 
Proof. (i) It has been shown that there is an $M^{\prime}$ such that the sequences $\left\{Y_{M}^{(m)}\right\}$ and $\left\{Z_{M}^{(m)}\right\}$ exist and are uniquely defined for $M \geqslant M^{\prime}$. To establish order $\mathbf{p}$ convergence, assume that $\left\|X_{M}^{(0)}-Y_{M}^{(0)}\right\|_{M}<K$ for all $M \geqslant M^{\prime}$. Let $U_{M}^{(m)}=Y_{M}^{(m)}-$ $Z_{M}^{(m)}$ and $V_{M}^{(m)}=X_{M}^{(m)}-Z_{M}^{(m)}$ for $m=0,1, \ldots, M$ and $M \geqslant M^{\prime}$. Now (2.1) and (2.2) give

$$
\begin{aligned}
& U_{M}^{(m)}=\mathbf{A} U_{M}^{(m-1)}-\mathbf{A} V_{M}^{(m-1)}+\frac{1}{M} \mathbf{B}_{1} W_{1 M}^{(m)}+\frac{1}{M} \mathbf{B}_{2} W_{2 M}^{(m)}, \\
& m=1,2, \ldots, M, M \geqslant M^{\prime}, \\
& W_{i M}^{(m)}=F_{i M}^{(m)}\left(Y_{M}^{(m)}\right)-F_{i M}^{(m)}\left(Z_{M}^{(m)}\right)=\int_{0}^{1} F_{i M}^{(m)^{\prime}}\left(Z_{M}^{(m)}+\tau U_{M}^{(m)}\right) d \tau U_{M}^{(m)}, \quad \\
& i=1,2 .
\end{aligned}
$$

Thus $W_{i M}^{(m)}=G_{i M}^{(m)} U_{M}^{(m)}$, where $\left\{G_{1 M}^{(m)}\right\}$ and $\left\{G_{2 M}^{(m)}\right\}$ are sequences of elements in $L\left(R^{N}, R^{N}\right)$ with $\left\|G_{1 M}^{(m)}\right\|_{1} \leqslant L_{1}$ and $\left\|G_{2 M}^{(m)}\right\|_{1} \leqslant L_{2}, m=1,2, \ldots, M, M \geqslant M^{\prime}$. Let $G_{M}^{(m)}=\mathrm{B}_{1} G_{1 M}^{(m)}+\mathrm{B}_{2} G_{2 M}^{(m)}$ so that $\left\|G_{M}^{(m)}\right\|_{1} \leqslant L_{1}\left\|B_{1}\right\|_{1}+L_{2}\left\|B_{2}\right\|_{1}=\beta$. Let $\mathbf{I}$ be the identity mapping in $L\left(R^{N}, R^{N}\right)$ and suppose that $M^{\prime}>\beta$. Then, for $M \geqslant M^{\prime}$, the inverse mappings $S_{M}^{(m)}=\left(I-(1 / M) G_{M}^{(m)}\right)^{-1}, m=1,2, \ldots, M$, exist and it follows that

$$
\begin{gathered}
U_{M}^{(m)}=S_{M}^{(m)} \mathbf{A} U_{M}^{(m-1)}-S_{M}^{(m)} \mathbf{A} V_{M}^{(m-1)} \\
U_{M}^{(m)}=S_{M}^{(m)} \mathbf{A} \cdots S_{M}^{(2)} \mathbf{A} S_{M}^{(1)} \mathbf{A} U_{M}^{(0)}-\sum_{i=1}^{m} S_{M}^{(m)} \mathbf{A} \cdots S_{M}^{(i+1)} \mathrm{A} S_{M}^{(i)} \mathbf{A} V_{M}^{(i-1)} \\
\left\|U_{M}^{(m)}\right\|_{M} \leqslant\left\|S_{M}^{(m)} \mathbf{A} \cdots S_{M}^{(2)} \mathbf{A} S_{M}^{(1)} \mathbf{A}\right\|_{M}\left\|U_{M}^{(0)}\right\|_{M} \\
+\sum_{i=1}^{m}\left\|S_{M}^{(m)} \mathbf{A} \cdots S_{M}^{(i+1)} \mathbf{A} S_{M}^{(i)} \mathbf{A} V_{M}^{(i-1)}\right\|_{M}
\end{gathered}
$$

(ii) Consider the expression $S_{M}^{(m)} \mathbf{A} \cdots S_{M}^{(2)} \mathrm{A} S_{M}^{(1)} \mathbf{A}$. Since

$$
S_{M}^{(r)}=\left(\mathrm{I}-\frac{1}{M} G_{M}^{(r)}\right)^{-1}=\mathrm{I}+\frac{1}{M} G_{M}^{(r)}+\frac{1}{M^{2}}\left(G_{M}^{(r)}\right)^{2}+\cdots
$$

the expression may be expanded in powers of $M$. There are $\left(\begin{array}{c}m+r-1 \\ r\end{array}\right)$ terms associated with $M^{-r}$ and a typical term is

$$
T_{r}=\frac{1}{M^{r}} \mathbf{A}^{\nu_{0}} G_{M}^{\left(i_{1}\right)} \mathbf{A}^{\nu_{1}} G_{M}^{\left(i_{2}\right)} \mathbf{A}^{\nu_{2}} \cdots G_{M}^{\left(i_{r}\right)} \mathbf{A}^{\nu_{r}}
$$

where $m \geqslant i_{1} \geqslant i_{2} \geqslant \cdots \geqslant i_{r} \geqslant 1$ and $\nu_{r} \geqslant 1$. Since the method is stable and hybrid, inequality (2.2) gives

$$
\left\|T_{r}\right\|_{M} \leqslant\left\|T_{r}\right\|_{1} \leqslant \alpha\left(\frac{\alpha \beta}{M}\right)^{r}, \quad M \geqslant M^{\prime}>\beta
$$


Since $\left(\begin{array}{c}m+r-1 \\ r\end{array}\right) \leqslant(\underset{r}{M+r-1})$, it follows that

$$
\begin{aligned}
\left\|S_{M}^{(m)} \mathbf{A} \cdots S_{M}^{(2)} \mathbf{A} S_{M}^{(1)} \mathbf{A}\right\|_{M} & \leqslant \alpha \sum_{i=0}^{\infty}\left(\begin{array}{c}
M+r-1 \\
r
\end{array}\right)\left(\frac{\alpha \beta}{M}\right)^{r} \\
& =\alpha\left(1-\frac{\alpha \beta}{M}\right)^{-M} \leqslant \alpha e^{2 \alpha \beta}
\end{aligned}
$$

provided that $M \geqslant M^{\prime}$ where $M^{\prime}>2 \alpha \beta$.

(iii) Consider an expression of the form $S_{M}^{(m)} \mathbf{A} \cdots S_{M}^{(2)} \mathrm{A} S_{M}^{(1)} \mathrm{A} V_{M}$, where

$$
\left\|V_{M}\right\|_{M}<k, \quad\left\|\mathrm{~A}^{\omega} V_{M}\right\|_{M}<\frac{k}{M}, \quad M=1,2,3, \ldots
$$

Again the expression may be expanded in powers of $M$, and a typical term associated with $M^{-r}$ is $T_{r} V_{M}$. Since the method is stable and hybrid,

$$
\begin{array}{ll}
\left\|T_{r} V_{M}\right\|_{M} \leqslant \alpha\left(\frac{\alpha \beta}{M}\right)^{r} k, & \nu_{r} \leqslant \omega, \\
\left\|T_{r} V_{M}\right\|_{M} \leqslant \alpha\left(\frac{\alpha \beta}{M}\right)^{r} \frac{k}{M}, & v_{r}>\omega .
\end{array}
$$

When $m>\omega$, the number of terms associated with $M^{-r}$ is

$$
\begin{aligned}
& \left(\begin{array}{c}
m+r-2 \\
r-1
\end{array}\right)+\left(\begin{array}{c}
m+r-3 \\
r-1
\end{array}\right)+\cdots+\left(\begin{array}{c}
m+r-1-\omega \\
r-1
\end{array}\right) \leqslant \omega\left(\begin{array}{c}
M+r-2 \\
r-1
\end{array}\right), \quad v_{r} \leqslant \omega, \\
& \left(\begin{array}{c}
m+r-2-\omega \\
r-1
\end{array}\right)+\left(\begin{array}{c}
m+r-3-\omega \\
r-1
\end{array}\right)+\cdots+\left(\begin{array}{c}
r-1 \\
r-1
\end{array}\right) \leqslant\left(\begin{array}{c}
M+r-1 \\
r
\end{array}\right), \quad v_{r}>\omega,
\end{aligned}
$$

and the results give

$$
\begin{aligned}
\left\|S_{M}^{(m)} \mathbf{A} \cdots S_{M}^{(2)} \mathbf{A} S_{M}^{(1)} \mathbf{A} V_{M}\right\|_{M} & \leqslant \frac{\alpha k}{M}(1+\omega \alpha \beta) \sum_{r=0}^{\infty}\left(\begin{array}{c}
M+r-1 \\
r
\end{array}\right)\left(\frac{\alpha \beta}{M}\right)^{r} \\
& \leqslant \frac{\alpha k}{M}(1+\omega \alpha \beta) e^{2 \alpha \beta},
\end{aligned}
$$

provided $m>\omega$ and $M \geqslant M^{\prime}$, where $M^{\prime}>2 \alpha \beta$. Since the method is order $\mathbf{p}$ consistent, this inequality gives

$$
\begin{aligned}
\sum_{i=1}^{m-\omega} \| S_{M}^{(m)} & \mathbf{A} \cdots S_{M}^{(i+1)} \mathbf{A} S_{M}^{(i)} \mathbf{A} V_{M}^{(i-1)} \|_{M} \\
& \leqslant(m-\omega) \frac{\alpha k}{M}(1+\omega \alpha \beta) e^{2 \alpha \beta} \leqslant \alpha k(1+\omega \alpha \beta) e^{2 \alpha \beta}
\end{aligned}
$$

Inequality (3.2) may be used now to give

$$
\sum_{i=1}^{m}\left\|S_{M}^{(m)} \mathrm{A} \cdots S_{M}^{(i+1)} \mathrm{A} S_{M}^{(i)} \mathbf{A} V_{M}^{(i-1)}\right\|_{M} \leqslant \alpha k(1+\omega \alpha \beta) e^{2 \alpha \beta}+\omega \alpha k e^{2 \alpha \beta}
$$

(iv) The bounds, applied to the inequality (3.1), give the result

$$
\left\|U_{M}^{(m)}\right\|_{M} \leqslant \alpha e^{2 \alpha \beta}\left(\left\|U_{M}^{(0)}\right\|_{M}+\omega k+k(1+\omega \alpha \beta)\right) .
$$

Order p convergence follows from $\left\|U_{M}^{(0)}\right\|_{M}=\left\|X_{M}^{(0)}-Y_{M}^{(0)}\right\|_{M}<K$ and from $\left\|X_{M}^{(m)}-Y_{M}^{(m)}\right\|_{M} \leqslant\left\|V_{M}^{(m)}\right\|_{M}+\left\|U_{M}^{(m)}\right\|_{M}$. 
4. Algebraic Conditions for Consistency. Certain algebraic conditions, for order $\mathrm{p}$ consistency of an additive $\left(A ; B_{1}, B_{2}\right)$ method, are obtained in this section. Since these conditions have a simple interpretation in terms of the order conditions for $(A, B)$ methods, the results generalize easily. The approach adopted is an extension of the technique used by Cooper [2]. Preliminary conditions for order $\mathbf{p}$ consistency are obtained by using bounds on the local truncation errors.

Lemma 1. An additive $\left(A ; B_{1}, B_{2}\right)$ method is order $\mathbf{p}(\omega)$ consistent with $\mathbf{p}=$ pe if and only if $A \mathrm{e}=\mathrm{e}$ and $\exists$ a diagonal matrix $C$ such that

$$
\begin{gathered}
\left\{C^{\tau}-A(C-I)^{\tau}-\tau B_{r} C^{\tau-1}\right\} \mathbf{e}=\mathbf{0}, \quad r=1,2, \tau=1,2, \ldots, p-1, \\
A^{\omega}\left\{C^{p}-A(C-I)^{p}-p B_{r} C^{p-1}\right\} \mathbf{e}=\mathbf{0}, \quad r=1,2 .
\end{gathered}
$$

Proof. Suppose that the conditions hold and define the consistency vector $\mathrm{c}$ by $\mathbf{e}_{i}^{T} \mathbf{c}=\mathbf{e}_{i}^{T} C \mathbf{e}_{i}, i=1,2, \ldots, s$. Consider the local truncation error

$$
E_{M}^{(m)}=X_{M}^{(m)}-\mathbf{A} X_{M}^{(m-1)}-\frac{1}{M} \mathbf{B}_{1} F_{1 M}^{(m)}\left(X_{M}^{(m)}\right)-\frac{1}{M} \mathbf{B}_{2} F_{2 M}^{(m)}\left(X_{M}^{(m)}\right) .
$$

Let $\mathbf{C}$ be the linear map defined by $C$ so that

$$
\begin{aligned}
& F_{r M}^{(m)}\left(X_{M}^{(m)}\right)= F_{r M}^{(m)}\left(X\left(\frac{m-1}{M} \mathrm{e}\right)\right)+\frac{1}{M} \mathbf{C} D F_{r M}^{(m)}\left(X\left(\frac{m-1}{M} \mathrm{e}\right)\right) \\
&+\frac{1}{2 M^{2}} \mathbf{C}^{2} D^{2} F_{r M}^{(m)}\left(X\left(\frac{m-1}{M} \mathrm{e}\right)\right)+\cdots \\
& X_{M}^{(m)}=X\left(\frac{m-1}{M} \mathrm{e}\right)+\frac{1}{M} \mathbf{C} X^{\prime}\left(\frac{m-1}{M} \mathbf{e}\right)+\frac{1}{2 M^{2}} \mathbf{C}^{2} X^{\prime \prime}\left(\frac{m-1}{M} \mathbf{e}\right)+\cdots
\end{aligned}
$$

where $X^{(\tau+1)}(\mathbf{w})=D^{\tau} F_{1 M}^{(m)}(X(\mathrm{w}))+D^{\tau} F_{2 M}^{(m)}(X(\mathrm{w}))$ for $\tau=0,1,2, \ldots$ Thus, since $\mathbf{p}=p \mathbf{e}$, the conditions give

$$
\left\|E_{M}^{(m)}\right\|_{M} \leqslant k^{\prime}, \quad\left\|\mathrm{A}^{\omega} E_{M}^{(m)}\right\|_{M} \leqslant \frac{k^{\prime}}{M},
$$

and the conditions are also necessary to establish these bounds. Let $V_{M}^{(m)}=X_{M}^{(m)}-$ $Z_{M}^{(m)}$ where $M \geqslant M^{\prime}$. Then

$$
\begin{aligned}
V_{M}^{(m)}= & E_{M}^{(m)}+\frac{1}{M} \mathbf{B}_{1}\left\{F_{1 M}^{(m)}\left(X_{M}^{(m)}\right)-F_{1 M}^{(m)}\left(Z_{M}^{(m)}\right)\right\} \\
& +\frac{1}{M} \mathbf{B}_{2}\left\{F_{2 M}^{(m)}\left(X_{M}^{(m)}\right)-F_{2 M}^{(m)}\left(Z_{M}^{(m)}\right)\right\}
\end{aligned}
$$

Choose $M^{\prime}>2 a \beta$, where $\beta=L_{1}\left\|B_{1}\right\|_{1}+L_{2}\left\|B_{2}\right\|_{1}$, and $a \geqslant\left\|A^{\nu}\right\|_{1}, \nu=0,1, \ldots, \omega$. Since $\mathbf{p}=p \mathbf{e}$, the Lipschitz conditions give

$$
\begin{gathered}
\left\|V_{M}^{(m)}\right\|_{M} \leqslant 2\left\|E_{M}^{(m)}\right\|_{M} \leqslant 2 k^{\prime}, \\
\left\|\mathbf{A}^{\omega} V_{M}^{(m)}\right\|_{M} \leqslant\left\|\mathbf{A}^{\omega} E_{M}^{(m)}\right\|_{M}+\frac{a \beta}{M}\left\|V_{M}^{(m)}\right\|_{M} \leqslant(1+2 a \beta) \frac{k^{\prime}}{M},
\end{gathered}
$$

and the method is order $p(\omega)$ consistent. Now suppose that the method is order $p(\omega)$ consistent for some consistency vector $\mathrm{c}$ and hence define $C$. Then the conditions are 
necessary, because

$$
\left\|E_{M}^{(m)}\right\|_{M} \leqslant \frac{3}{2}\left\|V_{M}^{(m)}\right\|_{M}, \quad\left\|\mathbf{A}^{\omega} E_{M}^{(m)}\right\|_{M} \leqslant\left\|\mathbf{A}^{\omega} V_{M}^{(m)}\right\|_{M}+\frac{a \beta}{M}\left\|V_{M}^{(m)}\right\|_{M} .
$$

Suppose that an $\left(A ; B_{1}, B_{2}\right)$ method is stable and order e $(\omega)$ consistent. Then an argument used by Cooper [2] may be applied, directly, to (4.2) to show that there exist $\mathrm{c}_{1}$ and $\mathrm{c}_{2}$ such that $A \mathrm{c}_{1}+B_{1} \mathrm{e}=\mathrm{c}_{1}+\mathrm{e}$ and $A \mathrm{c}_{2}+B_{2} \mathrm{e}=\mathrm{c}_{2}+\mathrm{e}$. Thus, the associated $\left(A, B_{1}\right)$ and $\left(A, B_{2}\right)$ methods are consistent. On the other hand, the lemma shows that, if these methods are consistent, the additive method is order $\mathbf{e}(\omega)$ consistent, if for some c,

$$
A^{\omega}(A-I)\left(\mathrm{c}_{1}-\mathrm{c}\right)=0, \quad A^{\omega}(A-I)\left(\mathrm{c}_{2}-\mathrm{c}\right)=0 .
$$

This is certainly true when $\mathrm{c}_{1}=\mathrm{c}_{2}$, but may also be true when $B_{1} \mathrm{e} \neq B_{2} \mathrm{e}$. The lemma also shows that, if the additive method is at least order $2 \mathbf{e}$ consistent, the condition $B_{1} \mathrm{e}=B_{2} \mathrm{e}$ must hold. That is, this condition must hold for any method which is order $\mathrm{p}$ consistent with $\mathrm{e}_{i}^{T} \mathrm{p} \geqslant 2$ for $i=1,2, \ldots, s$. Thus, methods with $B_{1} \mathbf{e} \neq$ $B_{2}$ e are comparatively difficult to obtain.

The lemma gives necessary and sufficient conditions for a method to be at least order $p$ e consistent, and these conditions imply that

$$
\left\|E_{M}^{(m)}\right\|_{1} \leqslant \frac{k}{M^{p}}, \quad\left\|V_{M}^{(m)}\right\|_{1} \leqslant \frac{k}{M^{p}},
$$

for some constant $k$. These bounds are used in the proof of the following theorem to give the order of magnitude of certain remainder terms.

THEOREM 2. Let $\mathbf{p}=\left(p_{1}, p_{2}, \ldots, p_{s}\right)^{T}$ be a given order vector with $p \leqslant p_{i} \leqslant 2 p, i=1,2, \ldots, s$, for some integer $p \geqslant 1$. An $\left(A ; B_{1}, B_{2}\right)$ method is order $\mathrm{p}(\omega)$ consistent if and only if $A \mathrm{e}=\mathrm{e}$ and there is a diagonal matrix $C$ such that, for $i=1,2, \ldots, s$,

$$
\begin{aligned}
& \mathrm{e}_{i}^{T} B_{r_{\mu}} C^{\tau_{\mu}-1} \cdots B_{r_{1}} C^{\tau_{1}^{-1}}\left\{C^{\tau_{0}}-A(C-I)^{\tau_{0}}-\tau_{0} B_{r_{0}} C^{\tau_{0}-1}\right\} \mathbf{e}=0, \\
& \tau_{0}+\tau_{1}+\cdots+\tau_{\mu} \leqslant p_{i}-1 \text {, } \\
& \mathbf{e}_{i}^{T} A^{\omega} B_{r_{\mu}} C^{\tau_{\mu}-1} \cdots B_{r} C^{\tau_{1}-1}\left\{C^{\tau_{0}}-A(C-I)^{\tau_{0}}-\tau_{0} B_{r_{0}} C^{\tau_{0}-1}\right\} \mathbf{e}=0, \\
& \tau_{0}+\tau_{1}+\cdots+\tau_{\mu} \leqslant p_{i}
\end{aligned}
$$

for $\mu=0,1,2, \ldots$, where $\tau_{0}, \tau_{1}, \tau_{2}, \ldots$, take all possible positive integer values and where $r_{\mu}=1,2$ for $\mu=0,1,2, \ldots$.

Proof. (i) Suppose that the conditions hold and suppose that a decomposition $f=f_{1}+f_{2}$ is used in step $m$ of an integration. Let $E=E_{M}^{(m)}$ be the local truncation error in step $m$ and let $V=X_{M}^{(m)}-Z_{M}^{(m)}$. Since the conditions imply that the method is at least order $p$ e consistent,

$$
F_{r}\left(Z_{M}^{(m)}\right)=F_{r}\left(X_{M}^{(m)}\right)-F_{r}^{\prime}\left(X_{M}^{(m)}\right) V+O\left(M^{-2 p}\right), \quad r=1,2 .
$$

Define $G^{\prime}=\mathbf{B}_{1} F_{1}^{\prime}\left(X_{M}^{(m)}\right)+\mathbf{B}_{2} F_{2}^{\prime}\left(X_{M}^{(m)}\right)$. Then (4.3) gives the relation $V=E+$ $(1 / M) G^{\prime} V+O\left(M^{-2 p-1}\right)$, and this may be used recursively to give 


$$
V=\left\{I+\frac{1}{M} G^{\prime}+\cdots+\frac{1}{M^{p}}\left(G^{\prime}\right)^{p}\right\} E+O\left(M^{-2 p-1}\right) .
$$

(ii) Each term in this expansion is treated separately, using the Taylor series

$$
\begin{gathered}
G^{\prime}=\sum_{\tau=0}^{\infty} \frac{1}{\tau ! M^{\tau}}\left[\mathbf{B}_{1} C^{\tau} D^{\tau} F_{1}^{\prime}(X)+\mathbf{B}_{2} C^{\tau} D^{\tau} F_{2}^{\prime}(X)\right] \\
E=\sum_{\tau=1}^{\infty} \frac{1}{\tau ! M^{\tau}}\left[\left\{\mathbf{C}^{\tau}-\mathbf{A}(\mathbf{C}-\mathbf{I})^{\tau}-\tau \mathbf{B}_{1} \mathbf{C}^{\tau-1}\right\} D^{\tau-1} F_{1}(X)\right. \\
\left.+\left\{\mathbf{C}^{\tau}-\mathbf{A}(\mathbf{C}-\mathbf{I})^{\tau}-\tau \mathbf{B}_{2} \mathbf{C}^{\tau-1}\right\} D^{\tau-1} F_{2}(X)\right]
\end{gathered}
$$

where $X=X(((m-1) / M)$ e). Let $X=x \oplus x \oplus \cdots \oplus x$ and let $\mathbf{W}$ be the linear map defined with respect to a real $s \times s$ matrix $W=\left\{w_{i j}\right\}$. Then

$$
F_{r}^{\prime}(X) \mathrm{W} F_{\rho}(X)=f_{r}^{\prime}(x) \sum_{j=1}^{s} w_{i j} f_{\rho}(x) \oplus \cdots \oplus f_{r}^{\prime}(x) \sum_{j=1}^{s} w_{s j} f_{\rho}(x)=\mathrm{W} F_{r}^{\prime}(X) F_{\rho}(X),
$$

so that a term $M^{-\mu}\left(G^{\prime}\right)^{\mu} E$ gives expressions of the form

$$
\begin{gathered}
\frac{1}{M^{\tau_{\mu}+\cdots+\tau_{1}+\tau_{0}}} \mathbf{B}_{r_{\mu}} \mathbf{C}^{\tau_{\mu}-1} \cdots \mathbf{B}_{r_{1}} \mathbf{C}^{\tau_{1}-1}\left\{\mathbf{C}^{\tau_{0}}-\mathbf{A}(\mathbf{C}-\mathbf{I})^{\tau_{0}}-\tau_{0} \mathbf{B}_{r_{0}} \mathbf{C}^{\tau_{0}-1}\right\} \\
\cdot D^{\tau_{1}-1} F_{r_{\mu}}^{\prime}(X) \cdots D^{\tau_{1}-1} F_{r_{1}}^{\prime}(X) D^{\tau_{0}-1} F_{r_{0}}(X) .
\end{gathered}
$$

Hence conditions (4.4) and (4.5) imply that

$$
\left\|M^{-\mu}\left(G^{\prime}\right)^{\mu} E\right\|_{M} \leqslant k, \quad\left\|M^{-\mu} \mathbf{A}^{\omega}\left(G^{\prime}\right)^{\mu} E\right\|_{M} \leqslant \frac{k}{M}
$$

for some constant $k$. Thus, $\|V\|_{M} \leqslant k$ and $\left\|\mathrm{A}^{\omega} V\right\|_{M} \leqslant k / M$ for some constant $k$, so that the conditions are sufficient for order $p(\omega)$ consistency.

(iii) Suppose the method is at least order pe consistent, where $p \geqslant 1$, and consider the expressions that arise from each term in the expansion for $V$. Since each expression involves a product of differing function and derivative elements, the given conditions are necessary.

The theorem may be extended, but there are further conditions to be taken into account. However, the conditions given in the theorem may be interpreted simply as the order conditions for an $\left(A, B_{1}\right)$ method, or for an $\left(A, B_{2}\right)$ method, plus all possible 'mixed' conditions that can arise if $B_{1}$ and $B_{2}$ may be interchanged. This is true in general.

In particular, the theorem gives conditions for $p=2$ and $\|\mathrm{p}\|_{1}=4$. Since $\|\mathrm{p}\|_{1} \geqslant 2$ and $B_{1} \mathrm{e}=B_{2} \mathrm{e}$ imply $p \geqslant 2$, the case $B_{1} \mathrm{e} \neq B_{2} \mathrm{e}$ may turn out to be of little interest. It may also be recalled that this case cannot be adapted to nonautonomous problems with arbitrary sequences of decompositions. For this case the theorem gives conditions only for $\|\mathrm{p}\|_{1} \leqslant 2$.

5. Examples of Additive Methods. The conditions for order $\mathbf{p}$ consistency cover a considerable variety of methods which may be represented by arrays of the form $\mathrm{p}|A| B_{1}\left|B_{2}\right| \mathrm{c}$, 


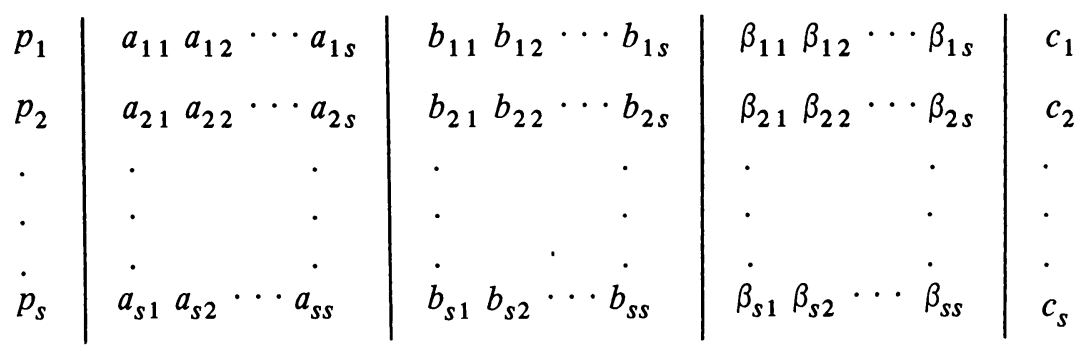

Such an array gives two $(A, B)$ methods which may be represented by the arrays $\mathbf{p}|A| B_{1} \mid \mathbf{c}_{1}$ and $\mathbf{p}|A| B_{2} \mid \mathbf{c}_{2}$. Only one example with $\mathbf{c}_{1} \neq \mathbf{c}_{2}$ is given here.

The following examples are intended to indicate some of the possibilities. Methods which are more competitive with existing procedures will be discussed in another article. It is also remarked that the efficacy of an additive method depends on the decompositions chosen. It is expected that the principal application of additive methods will be to handle stiff problems.

Order pe convergent methods are particularly easy to derive, since all such methods are hybrid, and Lemma 1 gives the only consistency conditions required. Indeed, any pair of stable linear multi-step methods of order $p$ yield an order $p$ e additive method. For example, the 3-step Adams-Moulton method and the 4-step AdamsBashforth method give the additive method described by the following array.

\begin{tabular}{|c|c|c|c|c|c|c|c|c|c|c|c|c|c|c|c|}
\hline 4 & $\begin{array}{ll}0 & 1\end{array}$ & 0 & 0 & 0 & 0 & 0 & 0 & 0 & 0 & 0 & 0 & 0 & 0 & 0 & -3 \\
\hline 4 & $\begin{array}{ll}0 & 0\end{array}$ & 1 & 0 & 0 & 0 & 0 & 0 & 0 & 0 & 0 & 0 & 0 & 0 & 0 & -2 \\
\hline & $\begin{array}{ll}0 & 0\end{array}$ & 0 & 1 & 0 & 0 & 0 & 0 & 0 & 0 & 0 & 0 & 0 & 0 & 0 & -1 \\
\hline & 00 & 0 & 0 & 1 & 0 & 0 & 0 & 0 & 0 & 0 & 0 & 0 & 0 & 0 & 0 \\
\hline & $\begin{array}{ll}0 & 0\end{array}$ & 0 & 0 & 1 & 0 & $\frac{1}{24}$ & $-\frac{5}{24}$ & $\frac{19}{24}$ & $\frac{9}{24}$ & $-\frac{9}{24}$ & $\frac{37}{24}$ & $-\frac{59}{24}$ & $\frac{55}{24}$ & 0 & 1 \\
\hline
\end{tabular}

This method may also be written in conventional linear multi-step form as

$$
\begin{aligned}
y_{m+4}= & y_{m+3}+\frac{h}{24}\left\{f_{1}\left(y_{m+1}\right)-5 f_{1}\left(y_{m+2}\right)+19 f_{1}\left(y_{m+3}\right)+9 f_{1}\left(y_{m+4}\right)\right\} \\
& +\frac{h}{24}\left\{-9 f_{2}\left(y_{m}\right)+37 f_{2}\left(y_{m+1}\right)-59 f_{2}\left(y_{m+2}\right)+55 f_{2}\left(y_{m+3}\right)\right\},
\end{aligned}
$$

and the method is inefficient, unless the decomposition $f=f_{1}+f_{2}$ is independent of the step. Such methods are order $\mathbf{p}(0)$ consistent and have $B_{1} \mathbf{e}=B_{2} \mathbf{e}$. When $\left\{f_{1}^{(m)}\right\}$ is a sequence of linear maps, these methods require the solution of one set of $n$ linear equations in each step.

Certain pairs of Runge-Kutta methods also yield additive methods. One example, already discussed in the introduction, may be represented by

$$
\begin{array}{l|lll|lll|lll|l}
2 & 0 & 0 & 1 & 0 & 0 & 0 & 0 & 0 & 0 & 0 \\
2 & 0 & 0 & 1 & 1 / 2 & 0 & 0 & 1 / 2 & 0 & 0 & 1 / 2 \\
& 0 & 0 & 1 & 1 / 2 & 0 & 1 / 2 & 0 & 1 & 0 & 1
\end{array}
$$


and is order $\mathbf{p}(1)$ consistent and may be applied to nonautonomous systems. The $\left(A, B_{1}\right)$ method is equivalent to the trapezoidal rule and is $A$-stable. Other examples of additive methods with $B_{1} \mathrm{e}=B_{2} \mathrm{e}$ are given by

$$
\begin{array}{l|llll|cccc|cccc|c}
3 & 0 & 0 & 0 & 1 & 0 & 0 & 0 & 0 & 0 & 0 & 0 & 0 & 0 \\
2 & 0 & 0 & 0 & 1 & \frac{1-\lambda}{2} & \frac{\lambda}{2} & 0 & 0 & 1 / 2 & 0 & 0 & 0 & 1 / 2 \\
2 & 0 & 0 & 0 & 1 & 2 \lambda+\mu-1 & 2(1-\lambda-\mu) & \mu & 0 & -1 & 2 & 0 & 0 & 1 \\
3 & 0 & 0 & 0 & 1 & \frac{1}{6} & \frac{2}{3} & \frac{1}{6} & 0 & \frac{1}{6} & \frac{2}{3} & \frac{1}{6} & 0 & 1
\end{array}
$$

and these methods are also order $\mathrm{p}(1)$ consistent. The $\left(A, B_{1}\right)$ method is $A$-stable when $\lambda=3 / 2$ and $\mu=5 / 6$. In this case the method may also be written as

$$
\begin{aligned}
y_{1}^{(m)}= & y_{3}^{(m-1)}-\frac{1}{4} h f_{1}^{(m)}\left(y_{3}^{(m-1)}\right)+\frac{3}{4} h f_{1}^{(m)}\left(y_{1}^{(m)}\right)+\frac{1}{2} h f_{2}^{(m)}\left(y_{3}^{(m-1)}\right), \\
y_{2}^{(m)}= & y_{3}^{(m-1)}+\frac{17}{6} h f_{1}^{(m)}\left(y_{3}^{(m-1)}\right)-\frac{8}{3} h f_{1}^{(m)}\left(y_{1}^{(m)}\right)+\frac{5}{6} h f_{1}^{(m)}\left(y_{2}^{(m)}\right) \\
& -h f_{2}^{(m)}\left(y_{3}^{(m-1)}\right)+2 h f_{2}^{(m)}\left(y_{1}^{(m)}\right), \\
y_{3}^{(m)}= & y_{3}^{(m-1)}+\frac{1}{6} h f\left(y_{3}^{(m-1)}\right)+\frac{2}{3} h f\left(y_{1}^{(m)}\right)+\frac{1}{6} h f\left(y_{2}^{(m)}\right),
\end{aligned}
$$

where a sequence of decompositions $\left\{f=f_{1}^{(m)}+f_{2}^{(m)}\right\}$ is used.

The final example is the additive method represented by the array

$$
\begin{array}{l|lll|lll|lll|l}
1 & 0 & 0 & 1 & 1 / 2 & 0 & 0 & 0 & 0 & 0 & 1 / 2 \\
2 & 0 & 0 & 1 & 0 & 1 / 2 & 0 & 1 & 0 & 0 & 1 / 2 \\
& 0 & 0 & 1 & 1 / 2 & 1 / 2 & 0 & 1 / 2 & 1 / 2 & 0 & 1
\end{array}
$$

which is order $\mathrm{p}(1)$ consistent. The $\left(A, B_{1}\right)$ method is equivalent to the implicit mid-point rule and is $A$-stable. In this example, $B_{1} \mathrm{e} \neq B_{2} \mathrm{e}$. In essence, information lost in the first stage is regained in the second stage.

School of Mathematical and Physical Sciences

University of Sussex

Brighton BN $19 \mathrm{QH}$, England

Department of Mathematics

College of Science

University of Baghdad

Baghdad, Iraq

1. J. C. BUTCHER, "On the convergence of numerical solutions to ordinary differential equations," Math. Comp., v. 20, 1966, pp. 1-10.

2. G. J. COOPER, "The order of convergence of general linear methods for ordinary differential equations," SIAM J. Numer. Anal., v. 15, 1978, pp. 643-661.

3. J. D. LAWSON, "Generalized Runge-Kutta processes for stable systems with large Lipschitz constants," SIAM J. Numer. Anal., v. 4, 1967, pp. 372-380.

4. R. D. SKEEL, "Analysis of fixed-stepsize methods," SIAM J. Numer. Anal., v. 13, 1976, pp. $664-685$. 